EBSJ is proud to present a selection of significant presentations made by investigators of the AOSpine North America community at the 11th Annual AOSNA Fellow meeting in Banff, Alberta. These studies demonstrate the bandwidth of research going on in AOSpine, ranging from basic sciences, population studies, and clinical outcomes projects to unprecedented, internationally linked research to solve major questions regarding surgical oncology. Congratulations to the authors and best wishes on their road to final publication!

\title{
Canine Notochordal Cell-Secreted Factors Protect Murine and Human Nucleus Pulposus Cells from Apoptosis by Inhibition of Activated Caspase-9 and Caspase-3/7
}

\author{
Arne Mehrkens ${ }^{1,2} \quad$ M. Zia Karim ${ }^{1} \quad$ Sarah Kim $^{1} \quad$ Raychel Hilario $^{1} \quad$ Michael G. Fehlings ${ }^{1}$ \\ William Mark Erwin ${ }^{1}$ \\ ${ }^{1}$ Toronto Western Research Institute, University of Toronto, Toronto, Canada \\ ${ }^{2}$ Department of Orthopaedic Surgery, Spine Unit, Basel University \\ Hospital, Basel, Switzerland, \\ Evid Based Spine Care J 2013;4:154-156. \\ Address for correspondence William Mark Erwin, DC, PhD, Toronto \\ Western Research Institute, Toronto Western Hospital, McLaughlin \\ Pavilion Room 11-408, 399 Bathurst Street, Toronto, ON, Canada \\ M5T2S8 (e-mail: mark.erwin@utoronto.ca).
}

\begin{abstract}
Keywords

- disc degeneration

- notochordal cells

- rescue
\end{abstract}

Introduction Effective therapies that may stop or even reverse disc degeneration remain elusive. A minimally invasive method through which nucleus pulposus (NP) cell viability could be achieved would revolutionize the treatment of degenerative disc disease (DDD). With the presented work, we have investigated if nonchondrodystrophic (NCD) canine intervertebral disc (IVD)-derived notochordal cell conditioned medium (NCCM) and chondrodystrophic (CD) canine IVD-derived conditioned medium (CDCM) are able to protect murine and human NP cells from apoptosis.

Materials and Methods We developed NCCM and CDCM from hypoxic culture of freshly isolated NPs from NCD and CD canines, respectively. We obtained murine NP cells from nine different C57BL/6 mice and human NP cells from four patients who underwent surgery for discectomy. The cells were cultured with ADMEM/F-12 (control media), NCCM, or CDCM under hypoxic conditions $\left(3.5 \% \mathrm{O}_{2}\right)$ and treated with IL$1 \beta+$ FasL or Etoposide. All media were supplemented with $2 \%$ fetal bovine serum. We then determined the expression of specific apoptotic pathways in the murine and human NP cells by recording activated caspase- 8 , caspase- 9 , and caspase-3/7 activity. Results In the murine NP cells, NCCM inhibits IL-1 $\beta$ + FasL- and Etoposide-mediated apoptosis via suppression of activated caspase- 9 and caspase-3/7, CDCM demonstrated an inhibitory effect on IL-1 $\beta$ + FasL-mediated apoptosis via caspase-3/7 (-Fig. 1A). In the human NP cells, NCCM inhibits Etoposide- mediated apoptosis via suppression of activated caspase-8, caspase-9, and mainly caspase-3/7. CDCM demonstrated an inhibitory effect on Etoposide-mediated apoptosis via suppression of activated caspase-8, caspase-9, and mainly caspase-3/7, though not as effective as NCCM (-Fig. 1B). Conclusion IL-1 $\beta$ + FasL are known key molecules in the progression of DDD. Here, we demonstrate that soluble factors secreted by the NCD IVD NP strongly protect murine received

May 9, 2013

accepted

July 18,2013 (c) 2013 Georg Thieme Verlag KG Stuttgart · New York
DOI http://dx.doi.org/ 10.1055/s-0033-1357363. ISSN 1663-7976. 
NP cells not only from IL-1 $1 \beta$ FasL but also from Etoposide-induced apoptosis via suppression of activated caspase- 9 and caspase-3/7. In the human samples, addition of $\mathrm{IL}-1 \beta+$ FasL did not increase cell death. Because the human cell samples were obtained from herniated discs that are probably already undergoing a degenerative process, it is likely that there was already some degree of activation by the endogenously secreted prodegenerative factors such as IL-1 $\beta$ + FasL. It may be that the NP cells, once they have reached a pivotal point of the degenerative cascade, no longer respond to exogenously applied IL-1 $\beta$ + FasL in contrast to the otherwise "healthy" discs obtained from the mice. Interestingly, the rescue effect of NCCM in the etoposide-treated cells (murine and human) suggests that NCCM is capable of influencing the signaling pathways known to be relevant to etoposide-induced cell death. A better understanding and harnessing of the restorative powers of the notochordal cell could lead to novel cellular and molecular strategies for the treatment of DDD.

A

Caspase 3/7 - murine NP cells

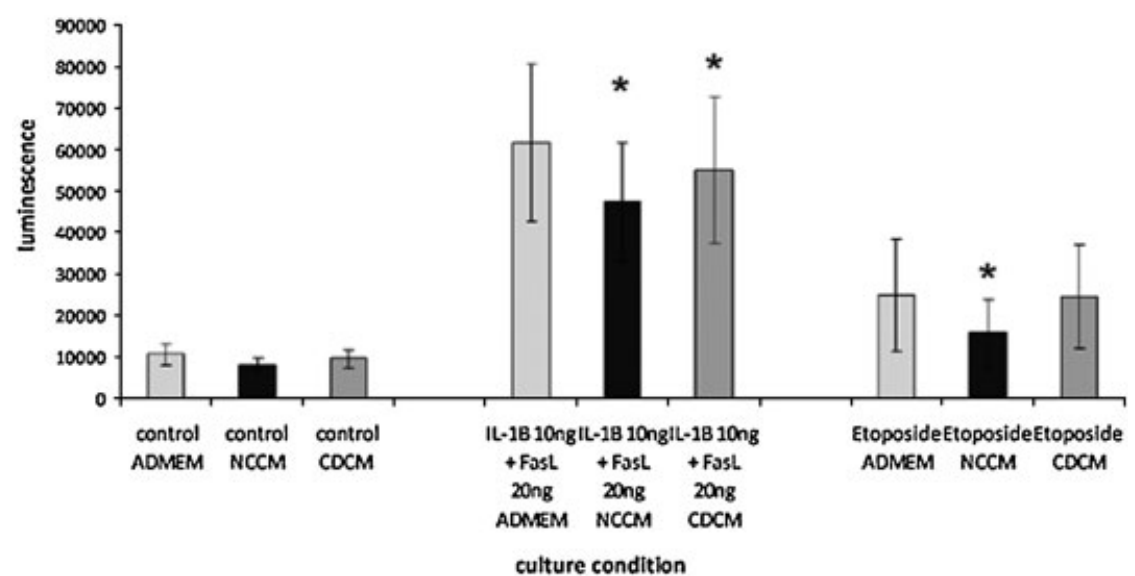

B

Caspase 3/7 - human NP cells

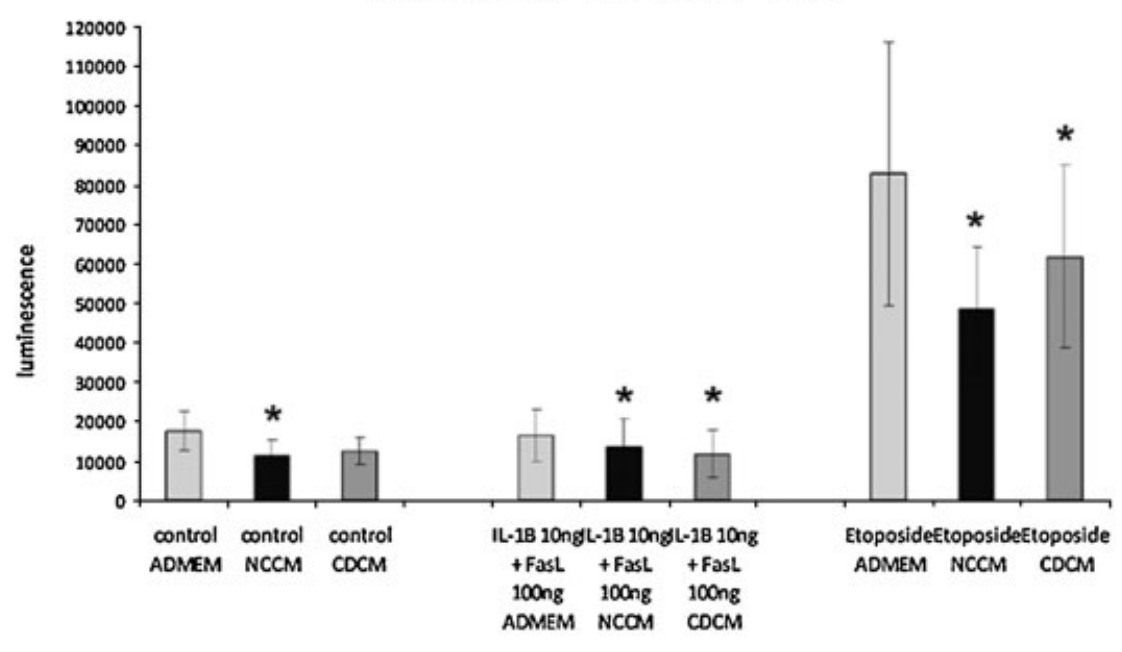

culture condition

Fig. 1 Assays for activated caspase 3/7 for murine (A) and human (B) cells. *Significant as compared with ADMEM in the same treatment group (control/IL-1 $\beta+$ FasL/etoposide). 


\section{Funding Sources}

1. AO Spine Europe and North America

2. The Caring Foundation

3. SGOT-Schweizer Gesellschaft fuer Orthopaedie und Traumatologie

4. Freie Akademische Gesellschaft Basel

5. Bangerter-Rhyner-Stiftung

6. North American Spine Society (Young Investigator award)

\section{Institutional Review Board}

The study was approved by Toronto University Health Network Research Ethics Board 11-0790-AE.

\section{Conflict of Interest}

None 